To appear in the International Journal of Computational Fluid Dynamics Vol. 00, No. 00, 00 Month 20XX, 1-20

\title{
Efficient preprocessing of complex geometries for CFD simulations
}

\author{
Zaib Ali ${ }^{a *}$, James Tyacke $^{\mathrm{b}}$, Rob Watson ${ }^{\mathrm{b}}$, Paul G. Tucker ${ }^{\mathrm{b}}$ and Shahrokh Shahpar \\ ${ }^{a}$ School of Mechanical and Manufacturing Engineering, National University of Sciences and Technology, \\ H-12, Islamabad, Pakistan; ${ }^{b}$ Department of Engineering, University of Cambridge, Cambridge, CB2 1PX, \\ $U K ;{ }^{c} R R$ Engineering Associate Fellow - Aerothermal Design System, Rolls-Royce, plc., Derby, England \\ DE24 $8 B J, U K$
}

\begin{abstract}
Preprocessing remains one the main bottlenecks in the Computational Fluid Dynamics (CFD) simulations of flows involving complex geometries as advances in the algorithm development, turbulence modeling and parallel computing are made. To this end, two approaches are presented here to efficiently deal with complex geometries in order to reduce the preprocessing time and manual effort. First, a hybrid blocking approach, combining the medial axis based method with level set iso-surface is presented to aid the block topology generation for subsequent structured meshing of complex 3D external flow domains. Secondly, a hierarchical geometry handling approach is demonstrated which makes use of the lower order modeling, overset meshes and zonal blocking for efficient preprocessing. Typical external aerodynamics cases have been showcased to describe how such techniques can be used to address modern challenges in the preparation of complex geometries for flow simulations.
\end{abstract}

Keywords: CFD Preprocessing; Multi-Block Structured Blocking ; Medial Axis Transform ; Wall Distance; Lower Order Modeling

\section{Introduction}

CFD is rapidly evolving as a design tool and is being increasingly utilized to simulate flow around complex 3D geometries typical of industrial scale. The CFD Vision 2030 Study (Slotnick et al. 2014) states that recent CFD advances have come from 'the use of larger meshes, more complex geometries, and more numerous runs'. However, preprocessing phase of the CFD run, which mainly involves domain decomposition and meshing, has remained one of the most time consuming steps for flow simulations and faces even bigger challenges as the geometric complexity grows. The CAD model can either be fully meshed using structured, unstructured and hybrid techniques or it could be represented by a combination of mesh and reduced order models.

There is a trade-off between the mesh quality, ease of generation, solver requirements and parallel mesh generation when choosing amongst the structured and unstructured mesh types. Unstructured meshes offer more flexibility for meshing complex 3D domains. Structured meshes, on the other hand, offer higher numerical accuracy and less storage than the unstructured meshes. They also allow relatively easy implementation of high-order finite volume and finite difference schemes e.g. compact schemes.

Multi-block structured mesh generation is among the most widely used meshing techniques in flow simulations. This is essentially a two-stage process. In the first stage, a suitable blocking topology is generated which divides the complex domain into simple sub-domains. The resulting blocks are subsequently meshed. This structured blocking offers an efficient meshing strategy for

*Corresponding author. Email: zaib.ali@smme.nust.edu.pk 
topologically simple configurations and standard templates exist for partitioning of such domains. However, the modern day design challenges demand the computational analysis of more realistic geometries. Aero-engine domains, for example, involving the fan, outlet guide vanes, gearbox shaft and nozzle coupled with wing, flap and pylon are now being used for flow simulations. Generation of block topology or domain decomposition of such multiply linked and more diverse geometries requires significant user intervention, or writing of templates as part of a library (Milli and Shahpar 2012). Thus, an automatic or semi-automatic blocking strategy can be beneficial to reduce the CFD design cycle time and could be a better alternative to the unstructured or hybrid meshing methods.

Fully automatic 3D block topology generation is a complex problem and currently there is no ideal block topology algorithm with all the desired features for structured mesh generation. However various automatic blocking approaches have been proposed with varying levels of automation and geometric complexity handling. This include approaches based on medial axis (Tam and Armstrong 1991; Price and Armstrong 1995a,b; Sheehy, Armstrong, and Robinson 1995), paving/plastering (Blacker and Myers 1993; Staten, Owen, and Blacker 2005) and more recently methods based on cross/frame field (Fogg, Armstrong, and Robinson 2015; Kowalski, Ledoux, and Frey 2014).

The medial axis transform (MAT) based algorithms for the domain decomposition have been presented in, for example, (Tam and Armstrong 1991; Price and Armstrong 1995a,b; Sheehy, Armstrong, and Robinson 1995). Here the medial axis is generated using the Voronoi based method. A subdivision is created resulting in one block for each medial vertex, medial edge and medial face. A midpoint subdivision is then used for meshing the blocks. An alternative has been presented by Rigby (Rigby 2004), called the 'TopMaker' approach, which makes use of medial vertices and parts of medial axis to block the domain. Medial vertices are defined as the points which are equidistant from three locations from the domain boundary. Consequently, six types of medial edges and appropriate rules are defined for creating the blocks. Further enhancements have been included to produce a good quality mesh, however this technique has yet to be extended for 3D.

Distance field based approaches are also widely used for the medial axis approximation and domain decomposition (Danielsson 1980; Ragnemalm 1993; Vleugels and Overmars 1995). A hybrid approach called differential MAT or d-MAT approach is presented in Xia and Tucker (Xia and Tucker 2010, 2011). Here, the hyperbolic-natured eikonal, level set equation is used to calculate the distance field (Tucker 2003). Medial axis point clouds are then extracted from the Laplacian or Hessian determinant of the distance field. A thinning algorithm is then used for thinning the point clouds into curves and surfaces. The method is illustrated in the Figure 1(a-c) for a simple domain. Such a hybrid approach thus avoids complexity of pure geometric approach and provides more accuracy than the pure image thinning algorithms. The differential equation based distance field and subsequent medial axis approximations have the advantage that a customized medial axis can be computed by manipulating the boundary conditions. This is shown in Figure 1(d) where the distance field relative to the part having the wall boundary condition is computed. This results in the simpler medial axis represented by the red curves in the Figure 1(f) thus avoiding triangular blocks encountered in, for example, Figure 1(c). In addition to the collision of wave fronts forming the compression shocks i.e. the medial axis, the expansion waves shown in the Figure 1(e) can be utilized to aid the blocking process. The expansion features are connected to the nearest 'medial vertex'; that is if that point lies within sight of the fan caused by the expansion feature. If not, it is connected to the nearest point on the shock feature topology (see green lines as Rule 1 in Figure $1(\mathrm{f})$ ). Rule 2 is borrowed from the TopMaker method (Rigby 2004); Hanging features (features which are not connected to anything) are extended to the nearest point on the geometry. This is shown by the green line in Figure $1(\mathrm{~g})$.

Malcevic (Malcevic 2011) presents another automated blocking strategy based on a Cartesian fitting method. While preserving the topology definition, a forward geometry simplification is performed followed by fitting the model into a Cartesian framework. The next step is blocking the domain after which the blocked model is mapped back on to the original geometry. Further operations such as removing singularities by J-grid wrapping are performed to enhance the block quality. This technique has been applied for blocking the end-wall cavities found in turbomachinery. 


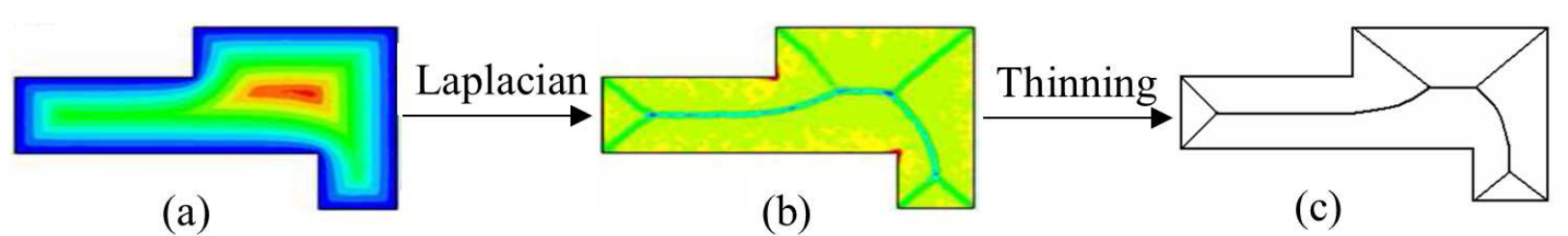

(a)
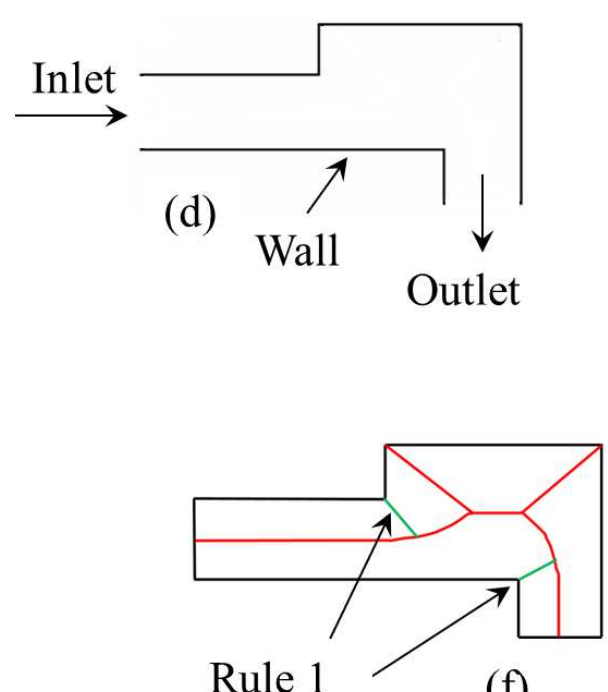

(f)

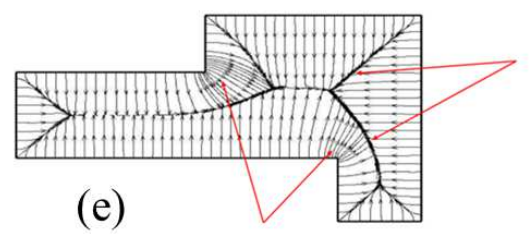

Expansion shock

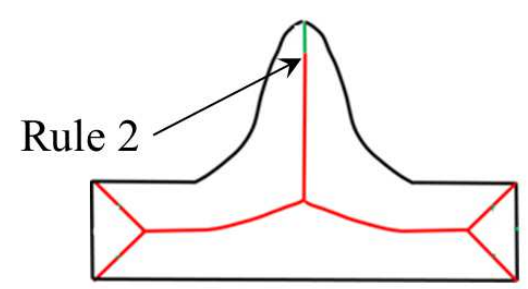

$(\mathrm{g})$
Compression shock

Figure 1.: d-MAT method and blocking; (a) distance field of a simple domain (b) medial axis extraction through the distance field Laplacian (c) point cloud thinning (d) simplified medial axis (e) shock and expansion features of the wave fronts (f) blocking rule 1 (g) blocking rule 2.

This technique is very simple but has only been demonstrated for $2 \mathrm{D}$ cases so far. The method sometimes produces some unnecessary mesh clustering across the block interfaces.

An assessment of various automatic block topology generation techniques surveyed above has been performed in (Ali and Tucker 2014; Ali 2015). The comparison has been carried out using an adjoint based error analysis of the meshes generated by these block topologies. It is found that, in general, the medial axis based approaches provide optimal blocking and yields better accuracy in computing the functional of interest. Mostly, domains having internal flows were used for this assessment. However, the medial axis based methods may not always yield an optimal block topology when dealing with complex 3D geometries and external flows. To overcome this limitation, a hybrid blocking technique is illustrated which makes use of the distance field isosurafce in addition to the medial axis transform. This is demonstrated using a wing-body-tail and a jet-wing-flap configurations. In addition to that, to reduce the blocking effort, a hierarchical geometry handling approach is also defined and applied to an engine-wing-flap configuration. These approaches are described next.

\section{Methodology}

\subsection{Novel hybrid blocking}

Consider an aero-engine jet-wing-flap (JWF) domain with a far-field as shown in the Figure 2. The medial axis close to the JWF geometry is shown in the enlarged view of $2 \mathrm{D}$ slice of domain in the Figure 2. Here the distance field and the corresponding medial axis is computed with respect to the geometry and the cylindrical far-field thus avoiding effect of the inlet and exit boundaries. The solid lines represent the shock features i.e. the medial axis and the dashed lines show the expansion features. Following this medial axis branches and even connecting the hanging and expansion 
features, a poor quality blocking would be achieved. This is also because, to generate the blocks, very small branches of the medial axis between the internal aeroengine geometry parts would have to be combined with very large branches between the aeroengine and the far-field.

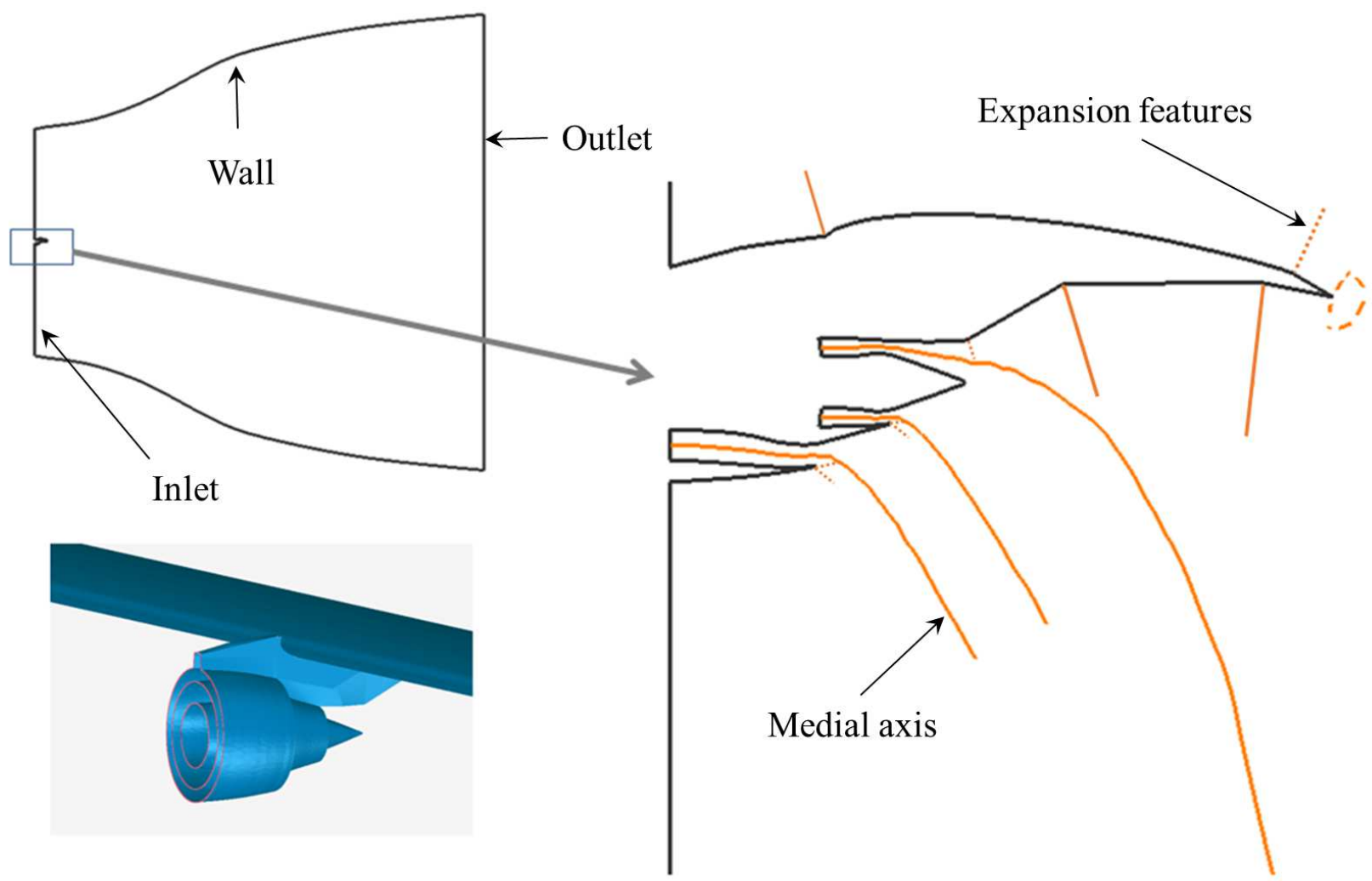

Figure 2.: Jet-wing-flap: medial axis transform (compression shock) and expansion features close to the geometry.

To overcome this limitation, the distance field function $d$ can be used. An isosurface (contour in 2D) of $d$ is wrapped around the geometry to facilitate the MAT based block topology generation. The wall distance computation is an intermediate step in the distance field based medial axis approximation and hence is available for use without any extra cost. Thus using an isosurface of the distance field instead of the farfield for the medial axis computation can significantly improve the medial axis based blocking. The hybrid blocking procedure is described below with the help of a simple 2D JWF geomtery. The extension of this methodology to the 3D cases also follows the same procedure as demonstrated later.

- The distance field is computed around the domain of interest as shown in the Figure 3(a). An exact equation for the wall distance $d$ is the hyperbolic eikonal equation which models the front propagation from the surface.

$$
F|\nabla v|=1+\Gamma \nabla^{2} v
$$

where $\Gamma \longrightarrow 0$ yielding viscosity solutions and the dependent variable $v$ describes the first arrival times of the wave fronts propagating from the boundaries. Here, $F(x)$ denotes the local speed function of these fronts and the wall distance in the $d=F v$ if $F$ is constant. The eikonal equation is solved using a fast marching method. The main steps of the procedure which is detailed in (Xia and Tucker 2010) are presented next. Substituting $\Gamma=0$ and taking 
square of Equation 1

$$
\nabla^{2} v=\frac{1}{F^{2}}
$$

The transport form of the above equation can be obtained by defining a pseudo-velocity vector $\mathbf{U}=\nabla v$ and adding a pseudo-time derivative $\frac{\partial v}{\partial \tau}$ which after rearranging the above equation, is given as

$$
\frac{\partial v}{\partial \tau}+\nabla(v \mathbf{U})-v \nabla \cdot \mathbf{U}=\frac{1}{F^{2}}
$$

This transport form of the equation is solved using the finite volume approach much like the other fluid flow equations. The integral form of the equation 3, for an arbitrary closed volume $\Omega$, can be written as

$$
\int_{\Omega} \frac{\partial v}{\partial \tau} d V+\oint_{\partial \Omega} v \mathbf{U} \cdot \mathbf{n} d A=\int_{\Omega}\left[\frac{1}{F^{2}}+f(v) \nabla^{2} v+g(v)\right] d V
$$

Here the viscous and extra terms i.e. $f(v)=v$ and $g(v)$ are set as zero to obtain the exact wall distance. The semi-discrete form of the Equation 4 can then be obtained as

$$
\frac{d v}{d \tau} \Delta V+\sum_{i}^{n f}\left(v U_{n}\right)_{i} \Delta A_{i}=S \Delta V
$$

where $\Delta V, \Delta A_{i}$ and $\mathrm{S}$ represent volume of the current control volume, the area of its ith face, and all terms on the right-hand side (source) of Equation 4 respectively. Also, $n f$ is the total number of faces. This discretization is then solved using the first-order upwind scheme and the time marching is performed using the multi-stage Runge-Kutta method. Moreover, to stabilize the iterative process, the velocity clipping and under-relaxation are applied (see (Xia and Tucker 2010)). Once the distance field is obtained, a suitable wall distance isosurface is extracted and exported to the mesh generator. This isosurface selection is currently arbitrary but it can be linked to a criteria. For example, the width of the shear layer regions in the jet wake can dictate this selection upstream or it could be based upon the dimensionless wall distance $y^{+}$value. The isosurface acts like a virtual geometry or a wrap around the real domain (see $3(\mathrm{~b}))$.

- The next step is approximation of the medial axis between the geometry and the distance field wrap. The Voronoi diagram based algorithm of Dey and Zhao (Dey and Zhao 2004) is used here for the medial axis approximation. This algorithm provides a more stable and continuous medial axis for complex 3D domains than the voxel thinning approach. The input to this program is the point cloud data of the geometry and the distance field isosurface. It makes use of the observation that certain Voronoi facets are positioned close to the medial axis if their dual Delaunay edges tilt away from the surface or are very long. Hence, the angle condition and the ratio condition are defined to filter such tilted and long Delaunay edges and the medial axis is approximated.

Let $V_{P}$ be the Voronoi diagram for a dense point set $P$ from a smooth compact surface $S \subset \mathbb{R}^{3}$. This Voronoi diagram is a cell complex comprising of Voronoi cells $V_{p_{p \in P}}$ and their facets, edges and vertices. Also, for each point $x \in S$, 


$$
V_{p}=x \in \mathbb{R}^{3} \mid\|p-x\| \leq\|q-x\|, \forall q \neq p
$$

where $p$ and $q$ are any two points in $P$. Let $D_{P}$ be the Delaunay triangulation of $P$ and dual to the Voronoi complex. The Delaunay triangles incident to point $p$ which are dual to the Voronoi edges intersected by a tangent plane at $p$ are used to construct the criteria. All the Delaunay edges that make relatively large angle with the planes of the triangles are filtered. If the angle between the vector $\mathbf{t}_{p q}$ from $p$ to $q$ and the normal $\mathbf{n}_{p t u}$ to a triangle $p t u$ is less than a threshold angle $\frac{\pi}{2}-\theta$ for all the triangles, then that associated Delaunay edge is filtered i.e.

$$
\max \angle \mathbf{n}_{p t u}, \mathbf{t}_{p q}<\frac{\pi}{2}-\theta
$$

where $0<\frac{\pi}{2}<\theta$. A value of $\theta=\frac{\pi}{8}$ gives good results. The ratio condition is based on the comparison of the length of the Delaunay edges with the circum-radii of the triangles. Thus those Delaunay edges are filtered which satisfy the criteria:

$$
\min \frac{\|p-q\|}{R}>\rho
$$

where $\|p-q\|$ defines the length of a Delaunay edge and $\rho$ is the circum-radius of a triangle $p t u$. A value of $\rho=8$ is normally used for dense point clouds. The medial axis is generated as a continuous surface which can be imported into the mesh generator. The medial axis for the JWF slice is shown in the Figure 3(c).

- To complete the blocking process, additional rules as described in Section 1 are manually used. Applying the rules, for example to the 2D JWF slice, the expansion features are connected to the nearest medial vertex or otherwise the medial axis as shown in the Figure 4. The same procedure is extended for $3 \mathrm{D}$ cases, where expansion surfaces are connected to the edges of the medial surfaces or otherwise the medial surfaces.

- Once the critical parts of the domain have been blocked using the medial axis, the far-field region can be partitioned using simple Cartesian fitting or H-type blocks. This is shown, for example, in Figure 4 with the green lines. This resulting domain decomposition is significantly better than the one obtained initially shown in the Figure 2. There can still be some regions where the block topology is unsatisfactory. Such areas must be manually altered. Hence, a semi automatic blocking process arises. The whole work flow along with approximate percentage of time consumed for various steps are shown in the Figure 5.

\subsection{Hierarchical geometry handling}

The blocking method presented in the last section clearly reduces the manual intervention and time consumption in preprocessing of the complex flow domains. However, the amount of user intervention and time consumption can further be reduced by using hierarchical combination of lower and higher order approaches. Such a combination is particularly useful for coupled set of complex geometries. This hierarchy is shown in the Figure 6 . At the bottom of this hierarchy is the smeared representation of the geometry through the use of the lower order methods such as immersed boundary method (IBM) and body force model (BFM) (Cao, Hield, and Tucker 2016; Cao et al. 2016). Next in the hierarchy is the real geometry resolved by the overset block topology based meshes which present a useful option for integrating various domains together without adding extra 


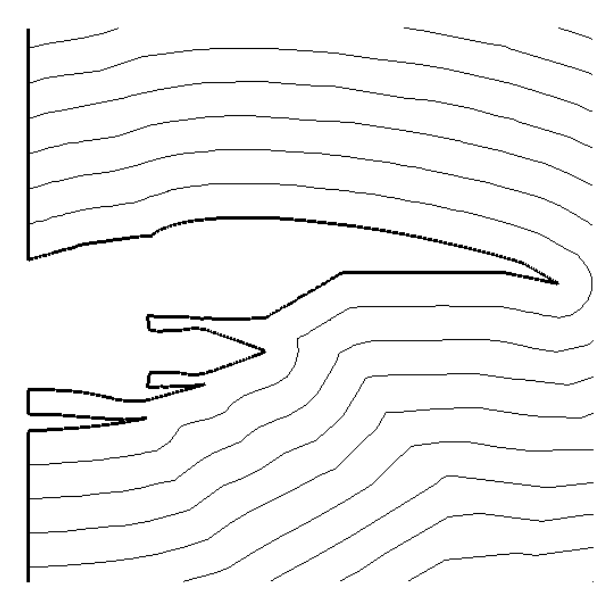

(a)

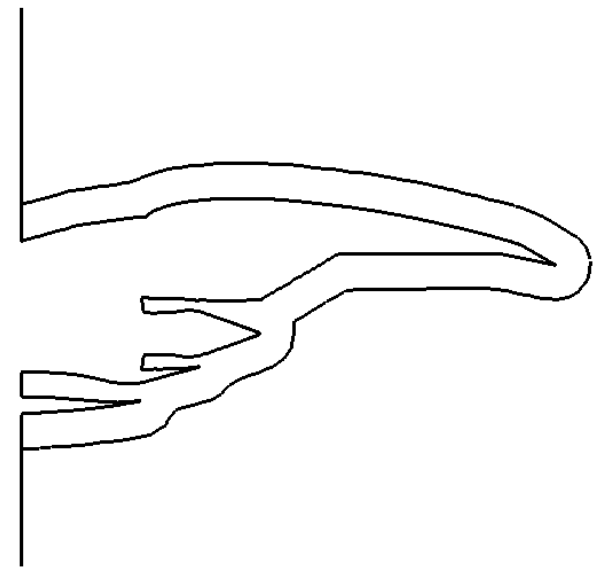

(b)

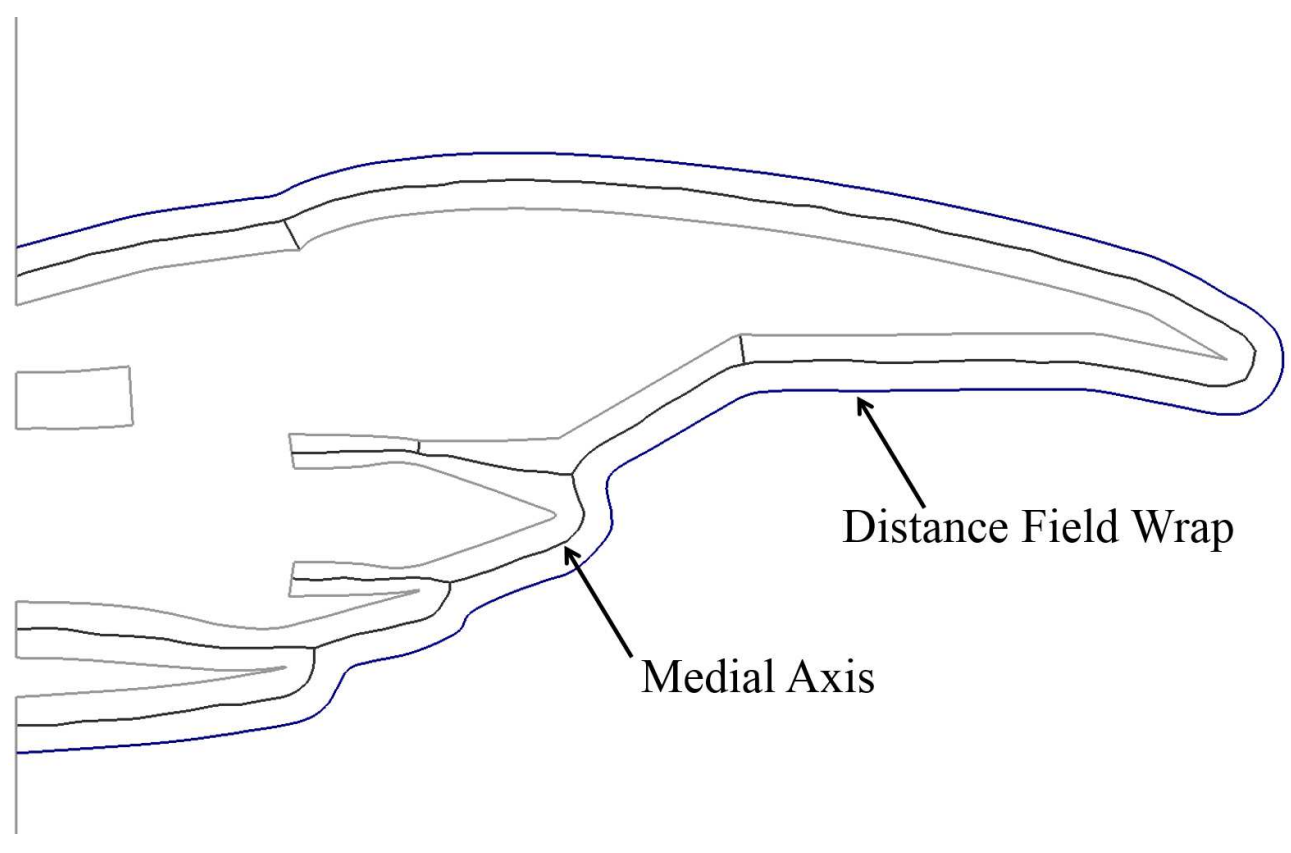

(c)

Figure 3.: Two dimensional jet-wing-flap geometry: (a) the distance field; (b) distance field wrap and (c) corresponding medial axis

complexity. The information between the overlapping parts is exchanged through interpolation. At the top is the real geometry resolved by body fitted and water tight blocking. Therefore, as a cost effective alternative to the fully blocked geometry, a combination of various high and low fidelity methods allows rapid addition and modeling of arbitrary geometry effects. This helps in reducing the design cycle time. The objective in this section is not to present any novel block topology generation method but to apply a zonal/hierarchical approach to handle complex domains in order to reduce the blocking effort (see Section 3.0.3). Such a hierarchical approach can be efficiently used to rapidly explore the design space and can aid the development of high fidelity numerical tools. 


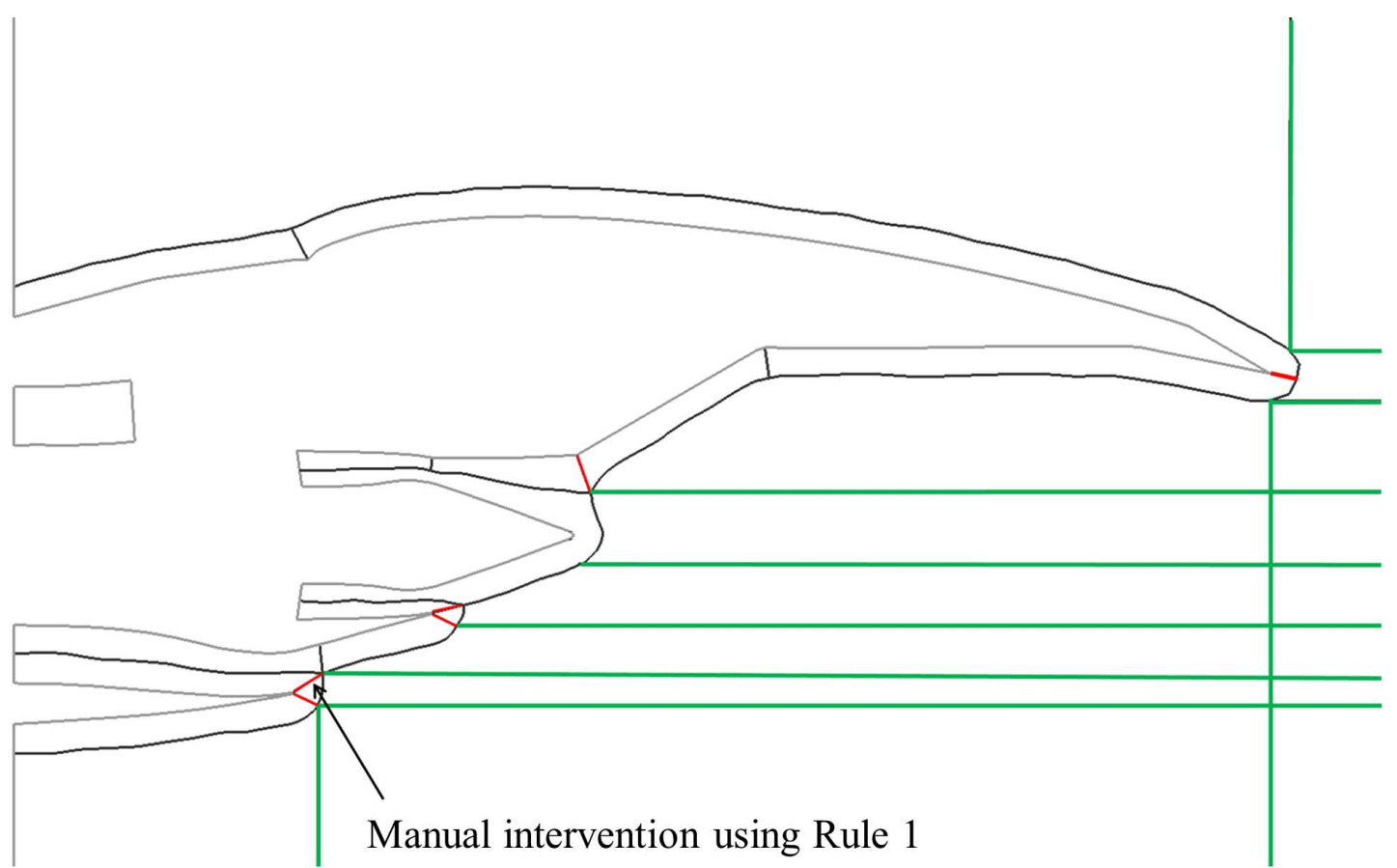

Figure 4.: Hybrid blocking around 2D geometry.

\subsubsection{Immersed Boundary Methods with optional filtered geometry (IBMfg)}

IBMfg (Immersed Boundary Methods with optional filtered geometry) is an effective lower order approach to model the effect of CAD geometries without explicit representation of real geometric features. The engine-wing-flap geometry case that is presented later employs a hierarchical geometry handling approach, and uses the IBMfg method to model the effect of the fan and outlet guide vanes. This hierarchical approach is outlined here.

The CFD program which was used for these calculations has two variants of an approach to the application of force fields. These fields can either be localized, with the objective of making the flow obey the no slip and impermeability conditions classical IBM or, alternatively, the force field can be consistent with assuming an infinite number of blades. The aerodynamic effects of a blade row are then modeled using an axisymmetric flow in each infinitesimal blade passage and, the required forces are added as source terms. These can either be expressed as parallel and normal components of these forces in a cylindrical coordinate system, or Cartesian coordinates can be used. Either way, the ultimate effect is to develop a force field that ensures the flow turns in a way consistent with the blade metal angles. It is as if the multiple blade geometries in the tangential flow direction have been subjected to a strong geometrical filtering. Hence, this more global approach is referred to here as IBM with filtered geometry IBMfg (see (Watson et al. 2017)).

Since the main focus of this work is preprocessing, these core solver numerical methods will not be dwelt on here further. For an overview of the approaches see Watson et al. However, as it is relevant to the core focus of the current work, we will discuss the geometry handling. For this, as is a standard industrial practice, the blade geometries are defined as point strings. These strings are used to define a surface mesh, and level sets are formed around this to define geometry. Hence, the implicit Euclidean distance based approach for blade geometrical modelling is ultimately used. This enables, for example, Boolean operations to be performed to make rapid geometric alterations (see (Dawes 2007)).

For the level sets internal to blades (the solid, not fluid geometry) the blade surface mesh is used in a Boundary Element Method (BEM) (see (Xia, Tucker, and Coughlin 2012; Watson et al. 2017)). This solves the linear Poisson equation below: 


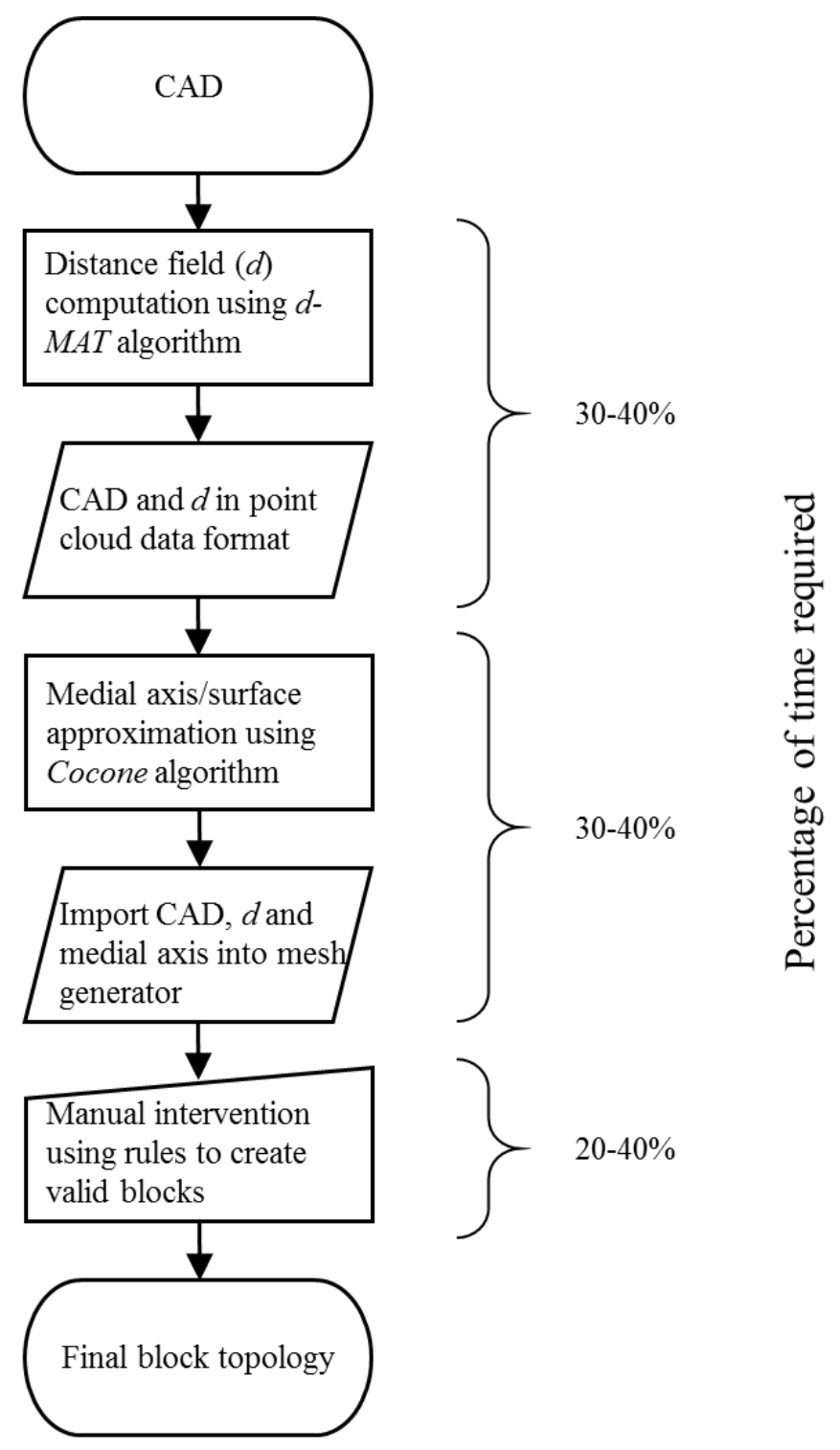

\section{Hybrid blocking process flow}

Figure 5.: Hybrid blocking work flow along with approximate percentage of time consumed for various steps.

$$
\nabla^{2} \phi=-1
$$

where $\phi$ is an auxiliary variable and $\phi(x)$ is a scalar function. Then nearest and furthest level sets from the interior walls are well approximated using the auxiliary analytical equation below:

$$
d= \pm \sqrt{\sum_{j=1,3}\left(\frac{\partial \phi}{\partial x_{j}}\right)^{2}}+\sqrt{\sum_{j=1,3}\left(\frac{\partial \phi}{\partial x_{j}}\right)^{2}+2 \phi}
$$

Note that the two roots of the equation correspond to the two noted wall distances. The sum of 


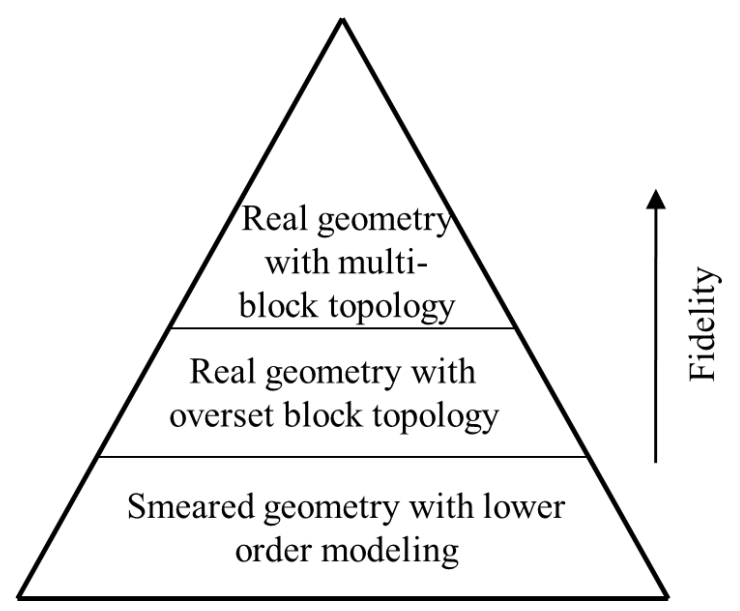

Figure 6.: Hierarchical geometry handling strategy.

these two values gives the local blade thickness. This is necessary for the CFD code to characterise the loss in flow area arising from the presence of the metal blade. The locus of $d+=d-$ gives the location of the blades medial axis, or camber line. This line defines the local flow angle that the blade is designed to impose on the flow. Hence, this approach supplies the only two ultimate geometrical inputs that are required by the IBMfg approach: the blade local angle, and the thickness of the blade normal to the mean flow direction in the relative frame. Aerodynamic losses arising from the full blade geometry need to be modeled, using for example, standard turbomachinery loss models that occur in low order throughflow models (see (Simon and Léonard 2007)).

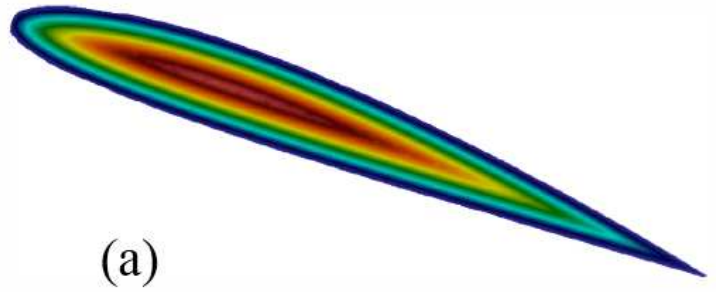

Figure 7.: Interior level sets and camber line preprocessing for IBMfg approach: (a) minimum Poisson wall distance, $d-$ (b) maximum Poisson wall distance, $d+$.

Figure 7 shows the approach applied to a basic aerofoil. Frame (a) gives the minimum wall distance, $d-$, and frame (b) gives the maximum wall distance, $d+$. As noted above, the sum of these two solutions gives the thickness distribution, and the line along which they are equal gives the camber line of the blade.

\section{Results}

\subsubsection{NASA CRM wing-body-tail}

In this section, the hybrid blocking is applied to partition the domain around a 3D NASA Common Research Model (CRM) horizontal wing-body-tail configuration. This model represents a modern, transonic and commercial aircraft designed to cruise at $M=0.85$ and $C_{L}=0.5$. The geometric and aerodynamic details about the model are described in (Vassberg et al. 2008, 2010). The configuration is shown in the Figure 8(a). The far-field is represented by a cube with boundaries ten fuselage lengths away upstream and downstream and ten spans away from the symmetry plane. First, the distance field wrap is computed around the model geometry which is shown in the Figure 


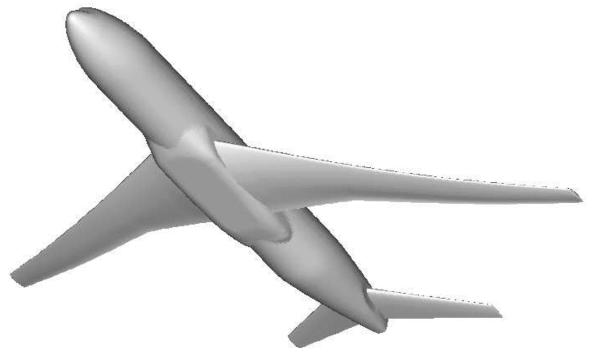

(a)

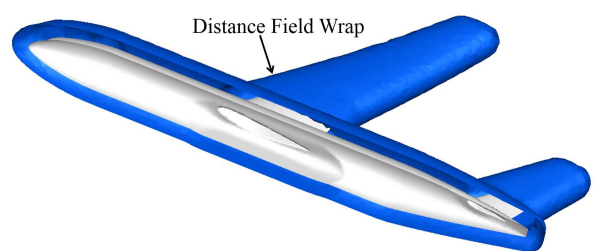

(b)

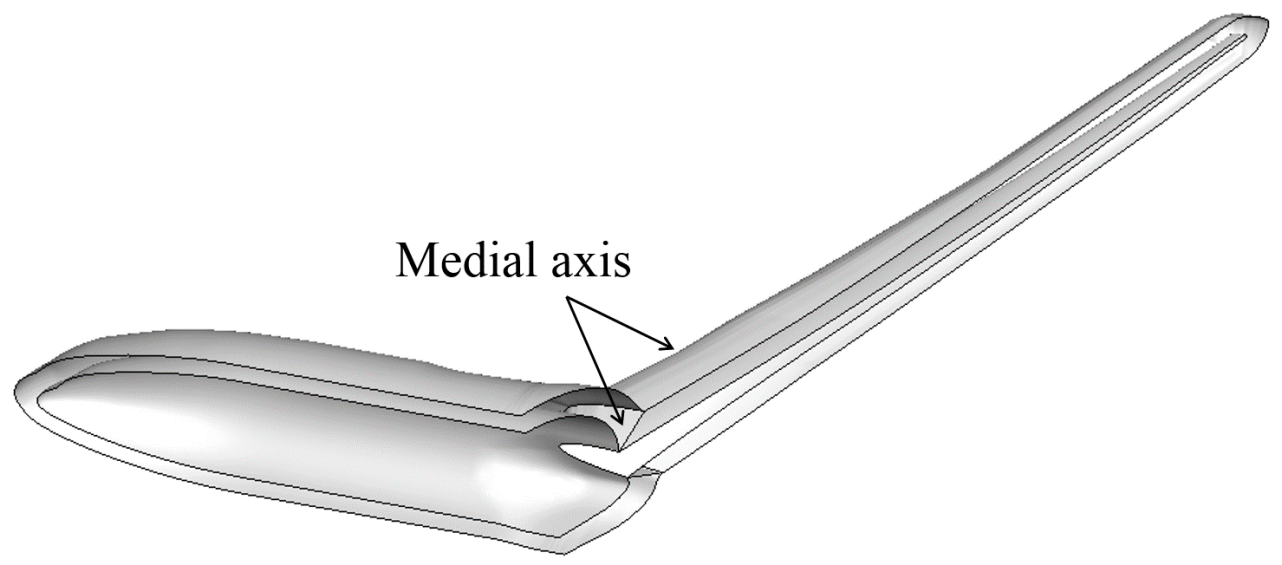

(c)

Figure 8.: NASA CRM: (a) wing-body-tail configuration (b) configuration with the distance field wrap (c) cut section with the medial axis.

$8(\mathrm{~b})$.

The medial axis is approximated around the model wrapped by the distance field isosurafce. A cut section of the model geometry and the medial axis is shown in the Figure 8(c). As can be seen, the medial axis branches at the wing-fuselage junction providing a useful block partitioning. The medial axis around the wing and tail also provides a block topology similar to O-type or C-type mesh blocks. To assist the blocking as shown in the Figure 9(a), expansion features at the trailing edges of the wing and the tail are joined to the nearest medial axis. After the blocking around the geometry is complete, the far-field domain partitioning is carried out. The region is partitioned to create a H-type mesh block. The block topology around the model is shown in the Figure 9(c). The volume and the surface mesh cuts are displayed in the Figure 9(d). The cell skewness histogram of the resulting mesh is shown in the Figure 9(b) where approximately $85 \%$ of the cells have skewness value below 0.55 (the ideal value being zero).

The NASA CRM configuration has been the test case for the 4th and 5th AIAA CFD drag prediction workshops (Vassberg et al. 2010; Levy et al. 2013). The aim of the workshop is to assess the state-of-the-art in the CFD methods for aircraft aerodynamic analysis. Here, we use the same flow conditions as given in the workshop to compute the flow around the test case. The simulations are performed in HYDRA which is an unstructured, finite volume, edge-based and compressible flow solver using MUSCL based flux differencing (Lapworth 2004; Moinier 1999).

The simulation is carried out at $M_{\infty}=0.85$ and $C_{L}=0.5$ with Reynolds number of $5 \times 10^{6}$ based on the reference chord length $C_{r e f}=7.00532 \mathrm{~m}$. Table 1 describes the free-stream flow conditions. A coarse mesh of approximately 4 million cells is used. The first grid node from the wall is located at $y^{+} \approx 1$. The SpalartAllmaras (SA) turbulence model is used for this simulation. The flow angle for this mesh to gain $C_{L}=0.5$ is $\alpha=2.36^{\circ}$. 


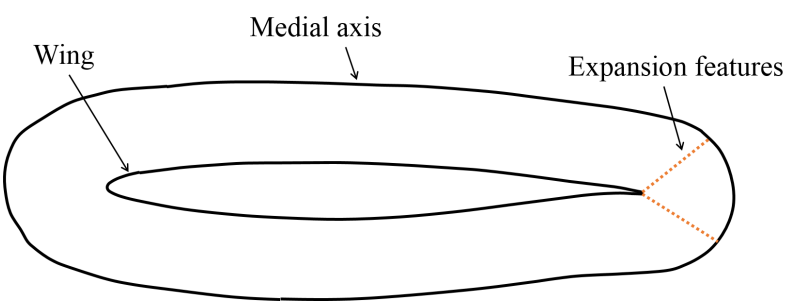

(a)

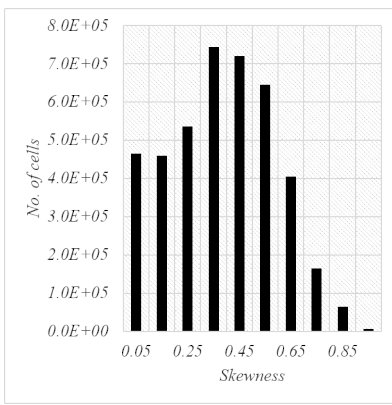

(b)

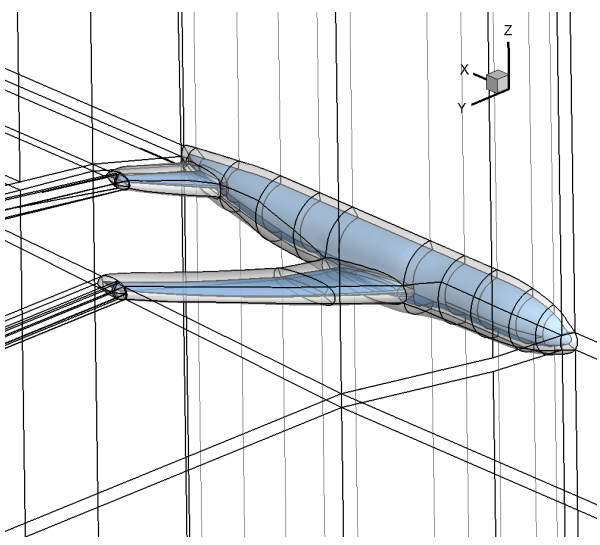

(c)

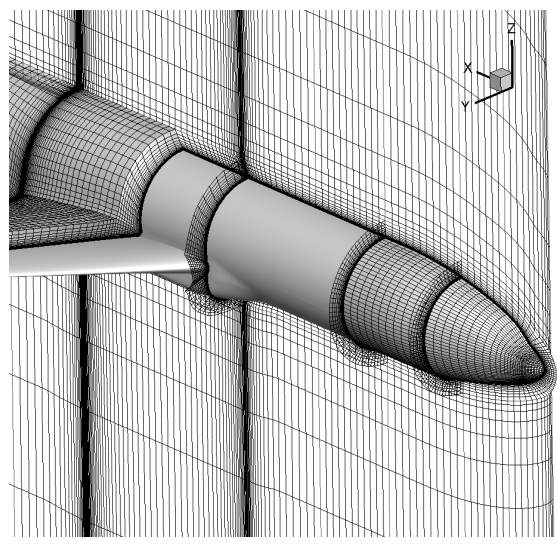

(d)

Figure 9.: NASA CRM wing-body-tail (a) blocking around the wing section (b) cell skewness histogram (c) hybrid blocking (d) mesh cut section.

\begin{tabular}{|l|l|}
\hline$v M_{\infty}$ & 0.85 \\
\hline$P_{\text {total }}$ & $201326.91 \mathrm{~Pa}$ \\
\hline$T_{\text {total }}$ & $310.93 \mathrm{~K}$ \\
\hline
\end{tabular}

Table 1.: NASA CRM free stream conditions.

The pressure coefficients at locations $13.06 \%, 28.3 \%$ and $50.24 \%$ of the wing span is shown in the Figure 10(a-c). The results are compared with the wind tunnel data at these locations. This experimental data is for $C_{L}$ values of 0.486 and 0.52 as shown in (Levy et al. 2013). A good level of agreement with the measurements can be observed in these plots.

\subsubsection{Jet-wing-flap}

In this section, the 3D jet-wing-flap case is presented. The geometry comprises of co-axial nozzle, pylon and a wing with a flap as shown in the Figure 11(a). This realistic aero-engine geometry has been used for detailed computational aero-acoustics analysis, as presented, for example in (Xia et al. 2012; Tyacke, Mahak, and Tucker 2015). The pylon adds complexity to the otherwise cylindrical nozzle topology along with the wing and the flap. Hence, blocking such a case demands significant user insight. After wrapping the distance field, the medial axis is approximated. This is shown in the Figure 11(b). To simplify the blocking procedure, small medial axis branches are removed for this case. This is followed by the inner blocking aided by the rules which is shown in the Figure 11(c).

The far-field domain decomposition can be then be carried out at this stage. However, one of the aims for the aero-acoustic jet simulations is to properly resolve the shear layers in the far-field. 


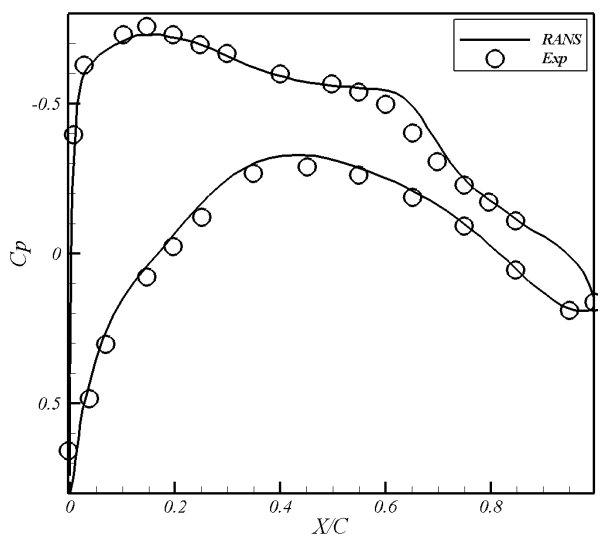

(a)

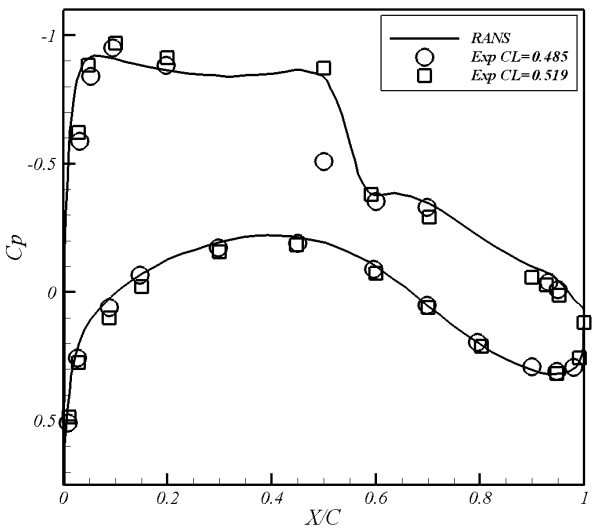

(c)

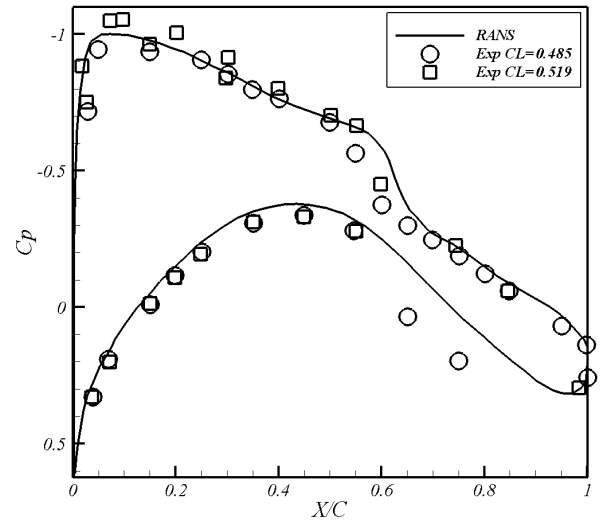

(b)

Figure 10.: NASA CRM wing-body-tail (a) pressure coefficients at 13.06\% (b) $28.3 \%$ and (c) $50.24 \%$ of the wing span.

This requires a good quality mesh aligned with the shear layer regions. The current block topology as shown in the Figure 11(c) is non-optimal for properly resolving the shear layers produced by the bypass and the core flows. Hence a manual alteration of the blocking around the pylon and the core flow exhaust is performed in which the high aspect ratio blocks are generated in the wake region by removing the medial surface surrounding the exhaust nozzle. The modified inner block topology with the far-field decomposition are shown in the Figure 12(a). Different cut sections of the resulting mesh (20 million cells) showing the surface and volume meshes are displayed in the Figures 12(c) and 12(d).

The cell skewness histogram is shown in the Figure 12(b) demonstrating that the mesh is of good quality. A RANS simulation using the SST $k-w$ is carried out on the mesh generated by the hybrid blocking. The first grid node from the wall is located at $y^{+} \approx 1$. The jet nozzle boundary conditions for this simulation are given in the Table 2 . The mean axial velocity contours at different axial planes are shown in the Figure 13. The results are compared with the experimental data and those obtained from the hybrid RANS-LES simulation performed in (Eastwood and Tucker 2011). Figure 14 shows an encouraging match where the axial velocity profiles in $z=0$ plane are plotted. Here, $U_{o}$ is the bypass exit velocity with value of approximately $300 \mathrm{~m} / \mathrm{s}$ and $D$ is the bypass jet diameter. The experimental data in these plots is for the clean nozzle jet without the effect of wing and flap, hence a scatter between the CFD and measurements can be seen.

The two cases presented above show how the hybrid blocking approach can be effectively used to decompose the complex geometries. The medial axis based domain decompositions also provide meshes having better flow alignment, as shown in (Ali and Tucker 2014; Ali 2015), when compared 


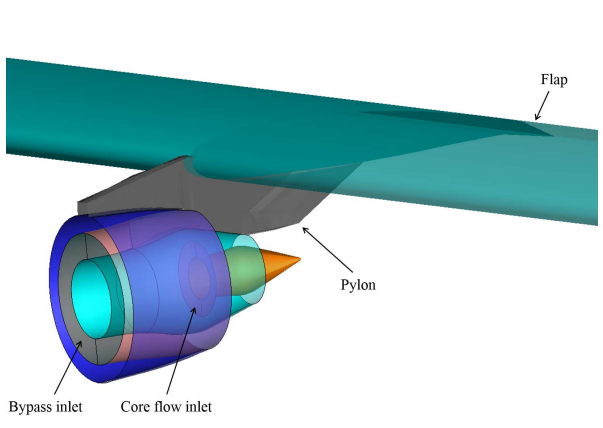

(a)

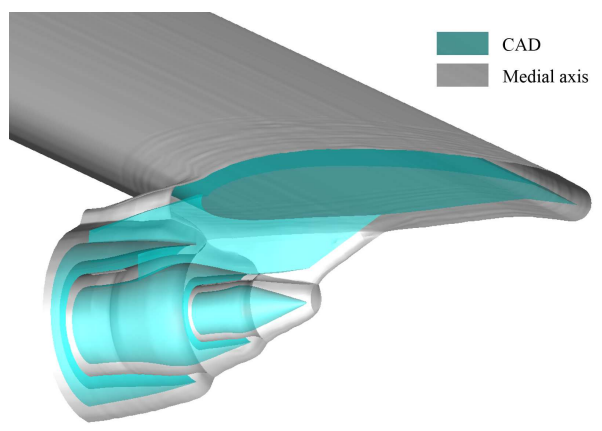

(b)

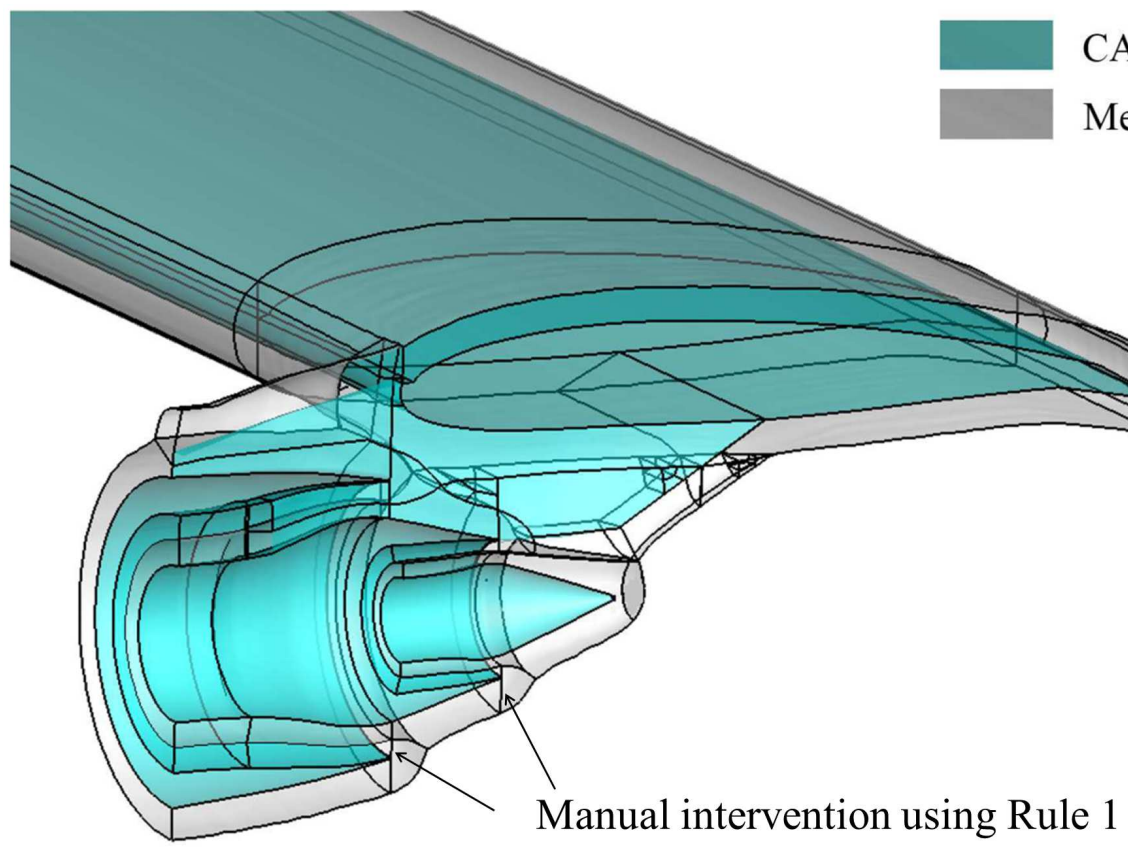

(c)

Figure 11.: Jet-wing-flap (a) CAD (b) CAD and the medial axis cut section (c) inner hybrid blocking.

\begin{tabular}{|l|l|l|}
\hline & Bypass & Core \\
\hline$P_{\text {total }}$ & $171,600 \mathrm{~Pa}$ & $164,000 \mathrm{~Pa}$ \\
\hline$T_{\text {total }}$ & $335.0 \mathrm{~K}$ & $889.0 \mathrm{~K}$ \\
\hline$\dot{m}$ & $9.325 \mathrm{Kgs}^{-1}$ & $1.633 \mathrm{Kgs}^{-1}$ \\
\hline
\end{tabular}

Table 2.: Jet nozzle boundary conditions.

to other partitioning methods e.g. Cartesian fitting and cross field based techniques. Hence, this technique further enhances the scope and applicability of these MAT based blocking methods. Also, the blocking templates could be generated using this approach which can speed up the mesh generation process and aid an inexperienced CFD user.

\subsubsection{Engine-wing-flap}

In the last section, a coaxial nozzle with pylon and wing geometry was presented which makes the rear or downstream part of the aero-engine. To carry out a more realistic simulation, the front 


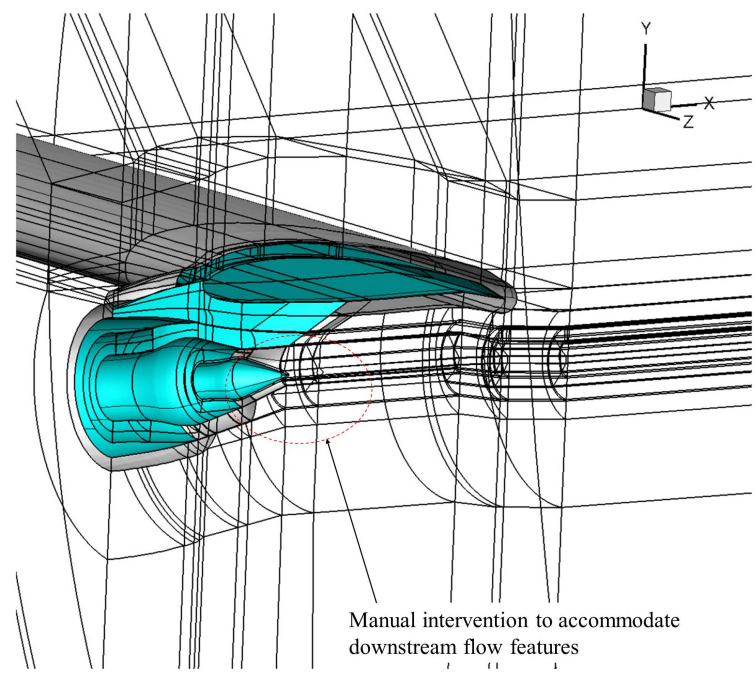

(a)

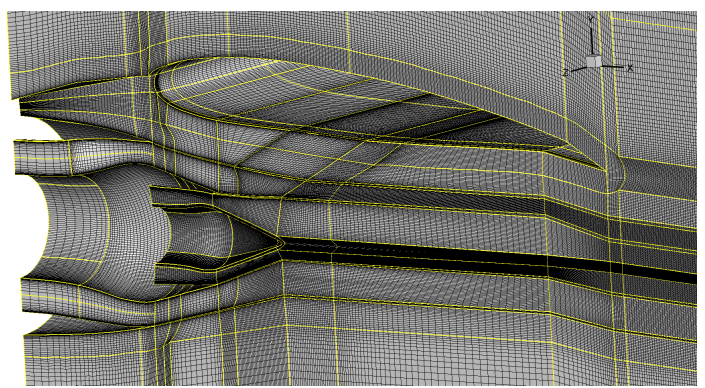

(c)

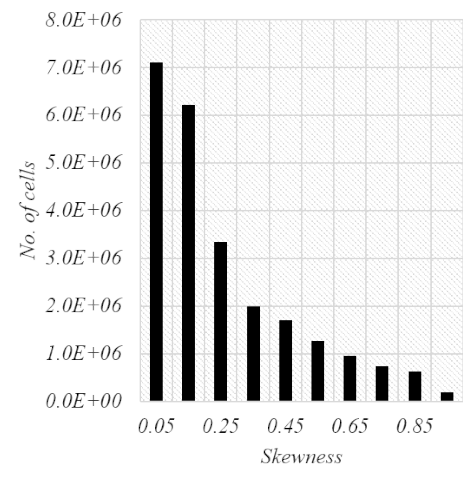

(b)

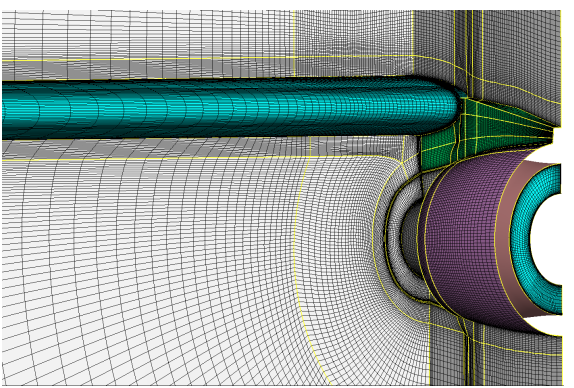

(d)

Figure 12.: Jet-wing-flap (a) modified inner blocking (to accommodate shear layers) with far-field block topology surface and volume mesh cut. (b) cell skewness histogram (c) mesh cut section at $z=0$ (d) a surface and volume mesh cut.

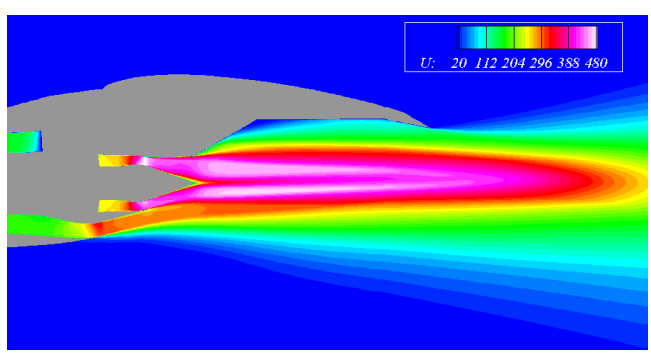

(a)

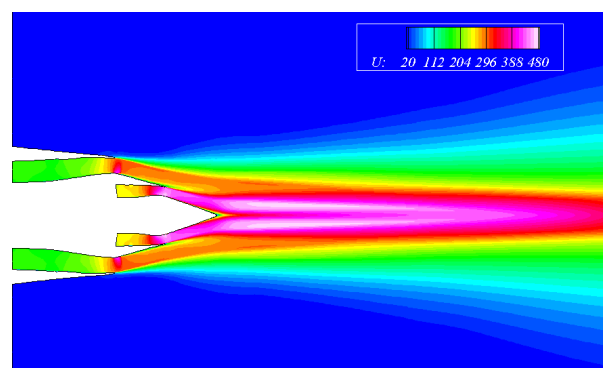

(b)

Figure 13.: Jet-wing-flap mean axial velocity contours; (a) $z=0$ plane (b) $y=0$ plane.

engine part containing the axis-symmetric intake, hub and splitter geometry is added to this rear part using the overset mesh at the interface. This procedure avoids re-blocking the domains to have a cell to cell match between the two zones. Also, a smeared fan geometry is used where the fan is modeled using the BFM (see the schematic in Figure 15(a)). The other downstream components are imprinted and treated again with the BFM but with localized sources. These internal geometry 


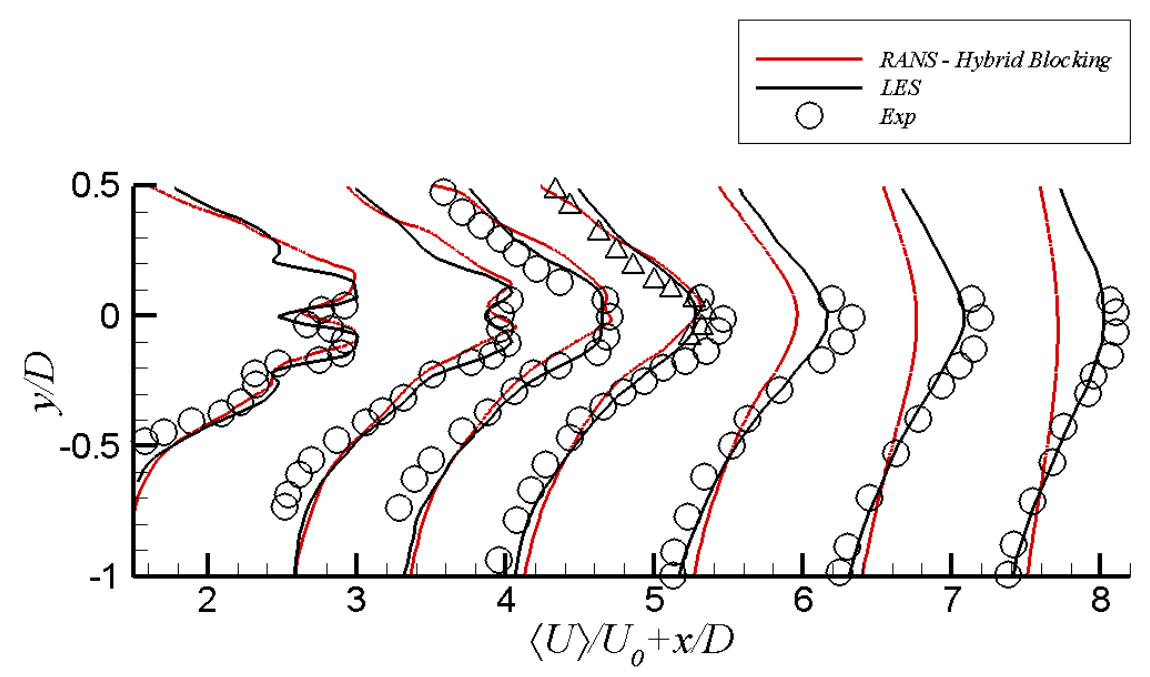

Figure 14.: Jet-wing-flap axial velocity profiles.

components include the downstream vanes, gearbox shaft, and the engine supporting A-frames. Thus using a hierarchical geometry handling approach, a complex domain can be readily meshed and analyzed for in a design optimization cycle.

The blocking and a coarse surface mesh for the configuration are shown in the Figure 16. A cut section of the mesh at $z=0$ plane is shown in the Figure 15(b). The total mesh size is 50 million. The cell skewness histogram for this integrated mesh is shown in the Figure 17(c). The internal geometry in the front part is modeled using a body force model (see section 2.2.1) which has been extended in (Loiodice, Tucker, and Watson 2010) to include the local blockage and wakes modeling. This is done by adding local enhanced source terms to generate wake zones, which is similar to adding the source terms in the IBM for simulating geometry or boundary on a non-conformal Cartesian mesh. A unified framework combining smeared geometry and the above mentioned crude IBM has been presented in (Cao, Hield, and Tucker 2016; Cao et al. 2016), which can be an area of future research.

Figure 17(a) shows the mean axial velocity contours at the $\mathrm{z}=0$ plane where the gear box shaft can be seen in the lower half of the bypass duct. Also, the fan and OGV zones can be seen in the Figure 17(b). The velocity profiles at $\mathrm{z}=0$ plane are shown in the Figure $17(\mathrm{~d})$. The plots are compared with the results obtained from the hybrid RANS-LES described in [134] where an acceptable match can be observed.

\section{Conclusions}

In the first part of the paper, a hybrid blocking method is demonstrated that makes use of the distance field isosurafce to act as a virtual geometry for the subsequent medial axis computation. Additional rules and the H-type blocking are employed to complete the block topology creation. This extends the original medial axis based method's ability to handle more complex geometries specially the external flows. Practical cases are included to demonstrate the use of this novel method. The short comings of the method for highly complex geometries are also described where manual alteration is required to resolve the important flow features. In the second part of the paper, an alternative to fully blocked geometry has been presented which handles the geometry by employing lower order modeling, hybrid blocking and the overset meshes. This provides an efficient and cost effective approach for addressing modern day meshing challenges and allows the rapid exploration of the design space by reducing the manual effort and time taken for preprocessing stage of the simulations. 


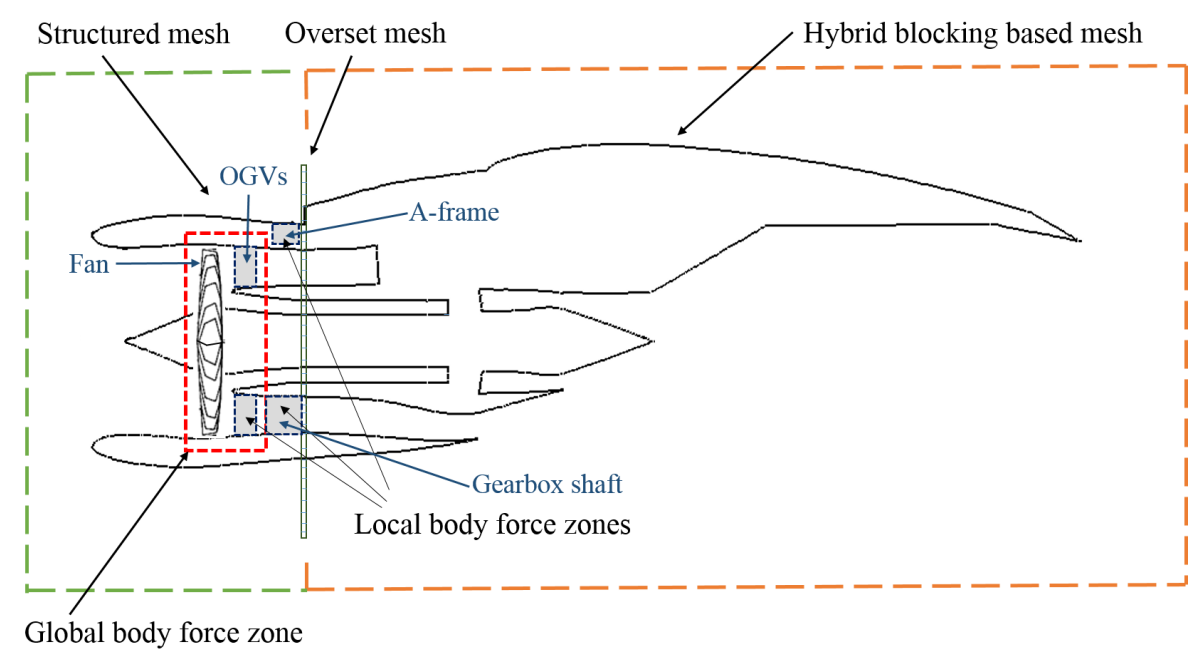

(a)

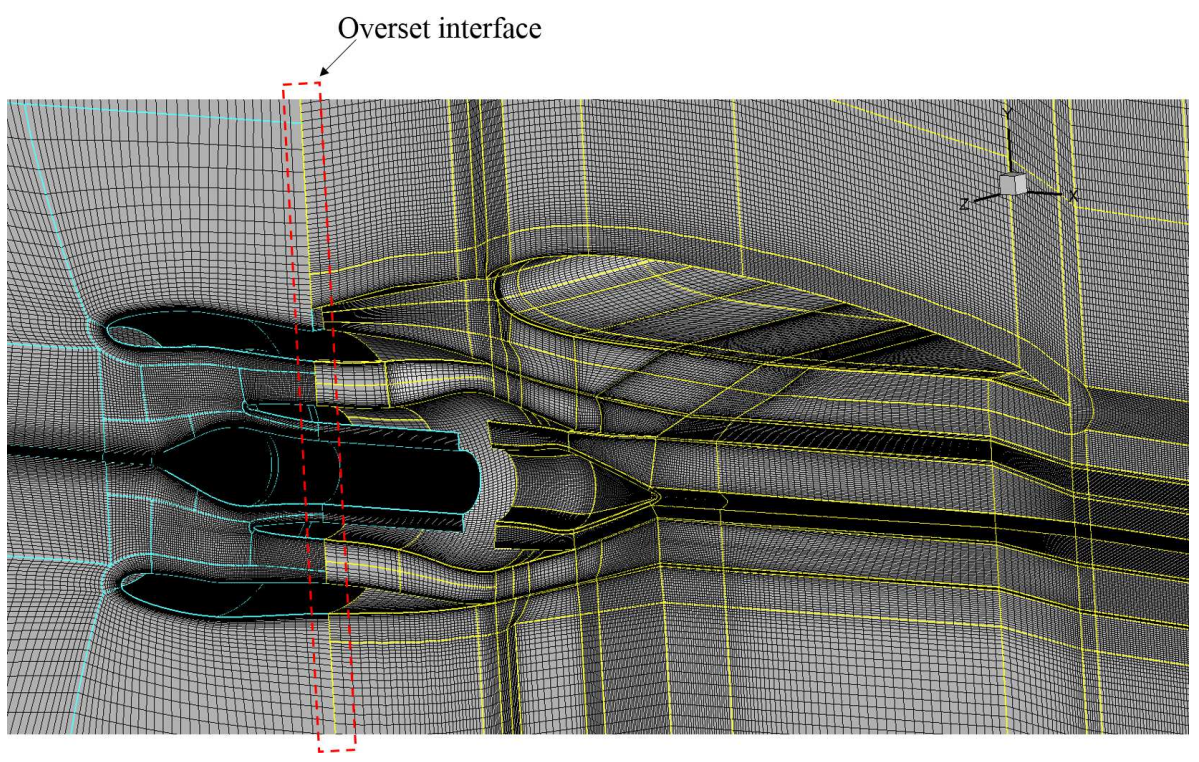

(b)

Figure 15.: Engine-wing-flap (a) schematics showing geometric zones and domains with different block topologies (b) cut section at $z=0$ plane.

\section{Acknowledgements}

The authors would like to thank the Higher Education Commission, Pakistan and the Rolls-Royce plc for the funding and permission to publish this work. Authors would also like to thank Dr. Caleb Dhanasekaran for providing the technical support and for the useful discussions.

\section{Funding}

Partial funding for this work was provided by Rolls-Royce plc under the grant TSB SILOET II TS/L00691X/1. 


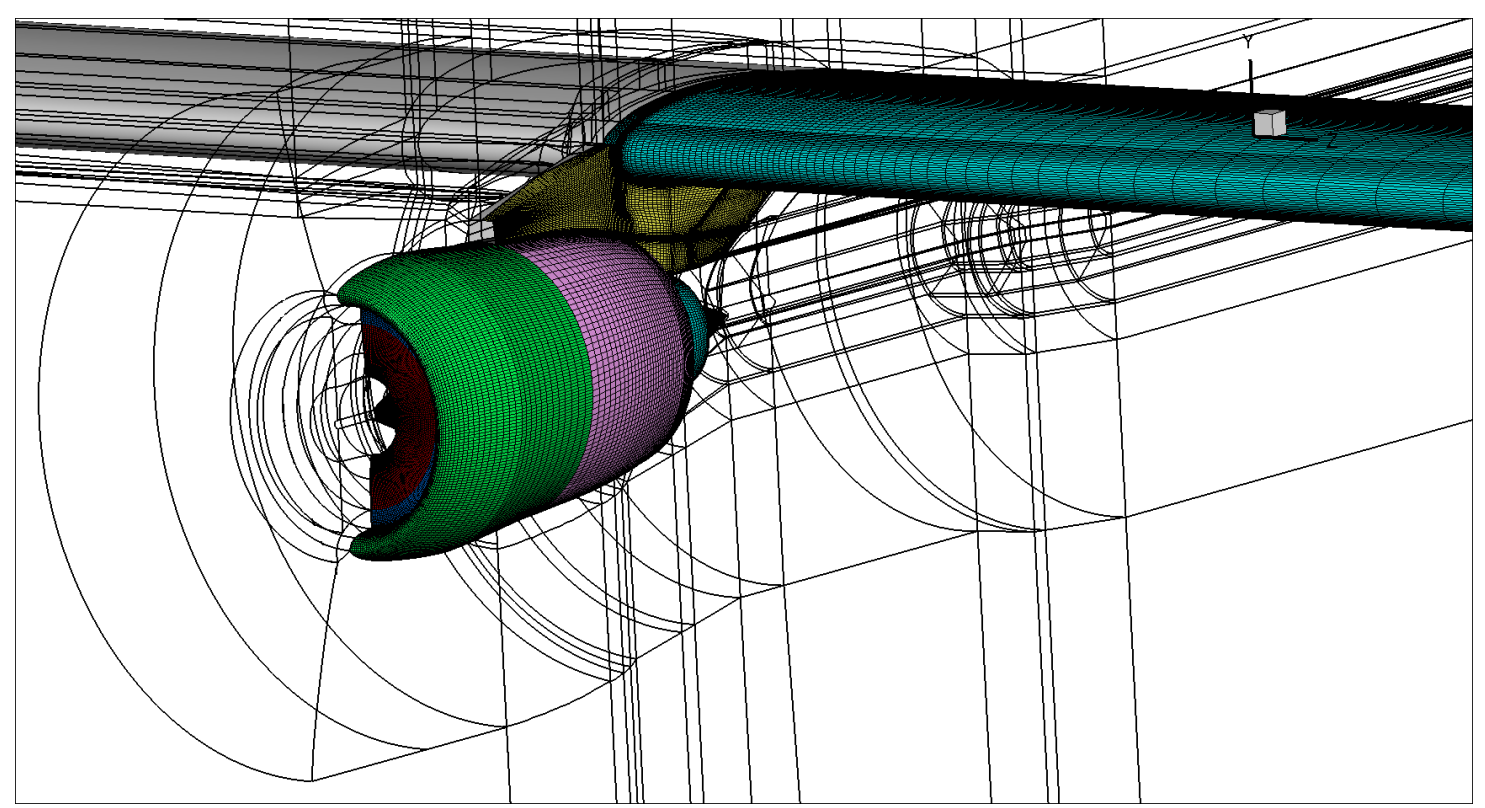

Figure 16.: Engine-wing-flap blocking and surface mesh.

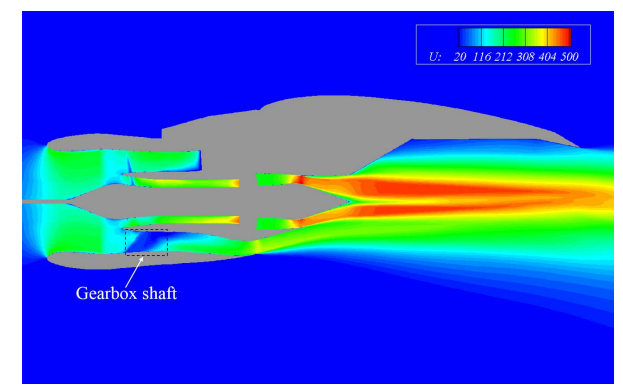

(a)

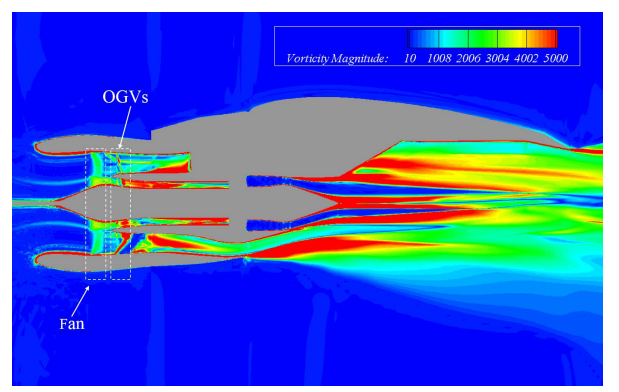

(b)

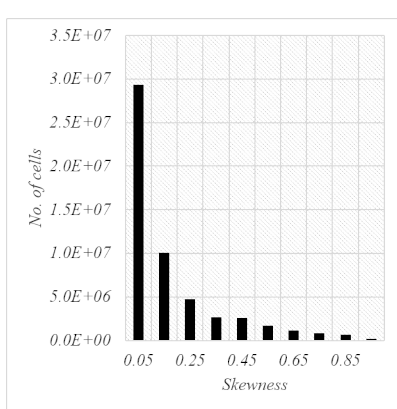

(c)

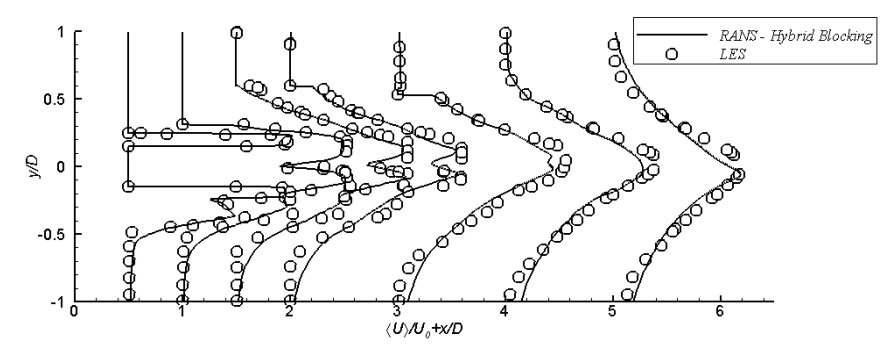

(d)

Figure 17.: Engine-wing-flap (a) $z=0$ plane, mean axial velocity contours. (b) $z=0$ plane, vorticity magnitude contours. (c) cell skewness histogram (d) downstream axial velocity profiles.

\section{References}

Ali, Z. 2015. "Optimal block topology generation for CFD meshing." Ph.D. thesis. Department of Engineeing, University of Cambridge, UK.

Ali, Z., and P. G. Tucker. 2014. "Multiblock structured mesh generation for turbomachinery flows." In Proceedings of the 22nd International Meshing Roundtable, 165-182. Springer. 
Blacker, T. D., and R. J. Myers. 1993. "Seams and Wedges in Plastering: A 3D Hexahedral Mesh Generation Algorithm." Engineering with Computers 2: 83-93.

Cao, T., P. Hield, and P. G. Tucker. 2016. "Hierarchical Immersed Boundary Method with Smeared Geometry." In 54th AIAA Aerospace Sciences Meeting, AIAA Science and Technology Forum and Exposition, 2016-2130.

Cao, T., N. R. Vadlamani, P. G. Tucker, A. R. Smith, M. Slaby, and C. T. J. Sheaf. 2016. "Fan-intake interaction under high incidence." In Proc. of ASME Turbo Expo, Seoul, South Korea. 13-17 June. ASME Paper Number GT2016-56561.

Danielsson, P-E. 1980. "Euclidean distance mapping." Computer Graphics and image processing 14 (3): $227-248$.

Dawes, W. N. 2007. "Turbomachinery computational fluid dynamics: asymptotes and paradigm shifts." Philosophical Transactions of the Royal Society of London A: Mathematical, Physical and Engineering Sciences 365 (1859): 2553-2585.

Dey, T. K., and W. Zhao. 2004. "Approximate medial axis as a Voronoi subcomplex." Computer-Aided Design 36 (2): 195-202.

Eastwood, S., and P. G. Tucker. 2011. "Hybrid LES-RANS of complex geometry jets." International Journal of Aeroacoustics 10 (5-6): 659-684.

Fogg, H. J., C. G. Armstrong, and T. T. Robinson. 2015. "Automatic generation of multiblock decompositions of surfaces." International Journal for Numerical Methods in Engineering 101 (13): 965-991.

Kowalski, N., F. Ledoux, and P. Frey. 2014. "Block-Structured Hexahedral Meshes for CAD Models using 3D Frame Fields." Procedia Engineering 82: 59-71.

Lapworth, L. 2004. "Hydra-CFD: a framework for collaborative CFD development." In International Conference on Scientific and Engineering Computation (IC-SEC), Singapore, June, Vol. 30.

Levy, D. W., K. R. Laflin, E. N. Tinoco, J. C. Vassberg, M. Mani, B. Rider, C. Rumsey, et al. 2013. "Summary of data from the fifth AIAA CFD drag prediction workshop." AIAA Paper No. AIAA-2013-0046.

Loiodice, S., P. G. Tucker, and J. Watson. 2010. "Modeling of Coupled Open Rotor Engine Intakes." In Proceedings of the 48th AIAA Aerospace Sciences Meeting and Exhibit, Orlando, FL, January, 4-7. AIAA Paper No. AIAA-2010-840.

Malcevic, I. 2011. "Automated Blocking for Structured CFD Gridding with an Application to Turbomachinery Secondary Flows." In 20th AIAA Computational Fluid Dynamics Conference, Honolulu, Hawaii.

Milli, A., and S. Shahpar. 2012. "PADRAM: Parametric design and rapid meshing system for complex turbomachinery configurations." In ASME Turbo Expo 2012: Turbine Technical Conference and Exposition, 2135-2148. American Society of Mechanical Engineers. ASME Paper No. GT 2012-69030.

Moinier, P. 1999. "Algorithm developments for an unstructured viscous flow solver." Ph.D. thesis. Oxford University.

Price, M.A., and C.G. Armstrong. 1995a. "Hexahedral Mesh Generation by Medial Surface Subdivision: Part I. Solids with Convex Edges." International Journal for Numerical Methods in Engineering 38: 33353359.

Price, M.A., and C.G. Armstrong. 1995b. "Hexahedral Mesh Generation by Medial Surface Subdivision: Part II. Solids with Flat and Concave Edges." International Journal for Numerical Methods in Engineering 38: 33353359.

Ragnemalm, I. 1993. "The Euclidean distance transform in arbitrary dimensions." Pattern Recognition Letters 14 (11): 883-888.

Rigby, D.L. 2004. ": A technique for automatic Multi-Block topology generation using the medial axis." NASA/CR FEDSM2003-45527.

Sheehy, D.J., C.G. Armstrong, and D.J. Robinson. 1995. "Computing the medial surface of a solid from a domain Delauney triangulation." In ACM Symposium on Solid Modeling Foundations and Applications, $201-212$.

Simon, J. F., and O. Léonard. 2007. "Modeling of 3-D losses and deviations in a throughflow analysis tool." Journal of Thermal Science 16 (3): 208-214.

Slotnick, Jeffrey, Abdollah Khodadoust, Juan Alonso, David Darmofal, William Gropp, Elizabeth Lurie, and Dimitri Mavriplis. 2014. "CFD vision 2030 study: a path to revolutionary computational aerosciences." .

Staten, M. L., S. J. Owen, and T. D. Blacker. 2005. "Unconstrained paving and plastering: A new idea for all hexahedral mesh generation." In Proceedings of 14th International Meshing Roundtable, 399416. Sandia National Lab.

Tam, T.K.H., and C.G. Armstrong. 1991. "2D Finite Element Mesh Generation by Medial Axis Subdivision." Adv. Eng. Software 13: 313324. 
Tucker, P.G. 2003. "Differential equation-based wall distance computation for DES and RANS." Journal of Computaional Physics 190(1): 229248.

Tyacke, J., M. Mahak, and P. G. Tucker. 2015. "LES of jet flow and noise with internal and external geometry features." AIAA Paper No. AIAA-2015-0503.

Vassberg, J.C., E. N. Tinoco, M. Mani, B. Rider, T. Zickuhr, D. Levy, O. P. Brodersen, et al. 2010. "Summary of the Fourth AIAA CFD Drag Prediction Workshop." AIAA Paper No. AIAA-2010-4547.

Vassberg, J. C., M. A. DeHaan, S. M. Rivers, and R. A. Wahls. 2008. "Development of a common research model for applied CFD validation studies." AIAA Paper No. AIAA-2008-6919.

Vleugels, J., and M. Overmars. 1995. "Approximating generalized Voronoi diagrams in any dimension." Technical report UU-CS-1995-14 Utretcht University.

Watson, R., J. Cui, Y. Ma, J. Tyacke, N. R. Vadlamani, M. F. Alam, Y. Dai, et al. 2017. "Improved Hierarchical Modelling for Aerodynamically Coupled Systems." In ASME Turbo Expo 2017. GT2017 June 2630, 2017, Charlotte, North Carolina, USA, American Society of Mechanical Engineers. ASME Paper No. GT2017-65225.

Xia, H., and P.G. Tucker. 2010. "Finite volume distance field and its application to medial axis transforms." International Journal for Numerical Methods in Engineering 82(1): 114134.

Xia, H., and P.G. Tucker. 2011. "Fast equal and biased distance fields for medial axis transform with meshing in mind." Applied Mathematical Modelling 35: 580419.

Xia, H., P. G. Tucker, and G. Coughlin. 2012. "Novel applications of BEM based Poisson level set approach." Engineering Analysis with Boundary Elements 36 (5): 907-912.

Xia, H., P. G. Tucker, S. Eastwood, and M. Mahak. 2012. "The influence of geometry on jet plume development." Progress in Aerospace Sciences 52: 56-66. 